\title{
Two disjoint shortest paths problem with non-negative edge length
}

$\operatorname{AUTHOR}(\mathrm{S})$ :

Kobayashi, Yusuke; Sako, Ryo

\section{CITATION:}

Kobayashi, Yusuke ...[et al]. Two disjoint shortest paths problem with non-negative edge length. Operations Research Letters 2019, 47(1): 6669

\section{ISSUE DATE:}

2019-1

URL:

http://hdl.handle.net/2433/244836

\section{RIGHT:}

(c) 2018. This manuscript version is made available under the CC-BY-NC-ND 4.0 license

http://creativecommons.org/licenses/by-nc-nd/4.0/; The full-text file will be made open to the public on 1 January 2021 in accordance with publisher's 'Terms and Conditions for Self-Archiving'.; この論文は出版社版でありません。引用の際 には出版社版をご確認じ利用ください。; This is not the published version. Please cite only the published version. 


\title{
Two Disjoint Shortest Paths Problem with Non-negative Edge Length
}

\author{
Yusuke Kobayashi \\ Kyoto University, Kyoto, Japan \\ Ryo Sako \\ University of Tsukuba, Tsukuba, Japan
}

\begin{abstract}
In the two disjoint shortest paths problem (2-DSPP), the input is a graph (or a digraph) and its vertex pairs $\left(s_{1}, t_{1}\right)$ and $\left(s_{2}, t_{2}\right)$, and the objective is to find two vertex-disjoint paths $P_{1}$ and $P_{2}$ such that $P_{i}$ is a shortest path from $s_{i}$ to $t_{i}$ for $i=1,2$, if they exist. In this paper, we give a first polynomial-time algorithm for the undirected version of the 2-DSPP with an arbitrary non-negative edge length function.
\end{abstract}

Keywords: disjoint paths, shortest path, polynomial-time algorithm

\section{Introduction}

The disjoint paths problem is one of the well-studied important problems in algorithmic graph theory and combinatorial optimization. In the problem, given a graph (or a digraph) $G=(V, E)$ and $k$ vertex pairs $\left(s_{1}, t_{1}\right), \ldots,\left(s_{k}, t_{k}\right)$, we find $k$ pairwise vertex-disjoint paths $P_{1}, \ldots, P_{k}$ where $P_{i}$ is a path from $s_{i}$ to $t_{i}$ for $i=1, \ldots, k$ (if they exist). If $k$ is a part of the input of the problem, then this is one of the classical NP-hard problems [5], and it remains NP-hard even if the input graph is constrained to be planar [6]. The disjoint paths problem is non-trivial even for the case with two terminal pairs. Indeed, the undirected version of the problem can be solved in polynomial time when $k=2[8,9,10]$, whereas the directed version is NP-hard even when $k=2$ [3]. For the case when the graph is undirected and $k$ is a fixed constant, Robertson and Seymour's graph minor theory gives a polynomial-time algorithm [7].

In this paper, we consider the problem of finding two disjoint paths in which each path has to be a shortest path. The problem is formally described as follows.

\section{Two Disjoint Shortest Paths Problem (2-DSPP)}

Input. A graph (or a digraph) $G=(V, E)$ with a length function $\ell: E \rightarrow \mathbb{R}_{+}$and two pairs of vertices $\left(s_{1}, t_{1}\right)$ and $\left(s_{2}, t_{2}\right)$ in $G$.

Find. Disjoint paths $P_{1}$ and $P_{2}$ such that $P_{i}$ is a shortest path from $s_{i}$ to $t_{i}$ for $i=1,2$, if they exist.

Here, $\mathbb{R}_{+}$denotes the set of non-negative real numbers. We can consider both directed and undirected variants of this problem, which we call the directed 2-DSPP and the undirected 2-DSPP,

Email addresses: yusuke@kurims.kyoto-u.ac.jp (Yusuke Kobayashi), s1820454@s.tsukuba.ac.jp (Ryo Sako) respectively. If the length of each edge is equal to zero, then this problem amounts to the two disjoint paths problem, which means that the 2-DSPP is a generalization of the two disjoint paths problem. With this observation, we can see that the directed 2-DSPP is NP-hard, because the two disjoint paths problem in digraphs is NP-hard [3]. It is interesting to note that the tractability of the problem changes if the length of each edge is restricted to be positive. Indeed, when the length of each edge is positive, Eilam-Tzoreff [2] devises an algorithm for the undirected 2-DSPP that is completely different from ones in $[8,9,10]$, and Bérczi and Kobayashi [1] give a polynomialtime algorithm for the directed 2-DSPP. The polynomial solvability of each case is summarized in Table 1. See [1] for other related results.

Table 1: Polynomial solvability of the 2-DSPP.

\begin{tabular}{lll} 
& Undirected & Directed \\
\hline$\ell=0$ (Disjoint Paths) & $\mathrm{P}[8,9,10]$ & NP-hard [3] \\
$\ell \geq 0$ & $\mathrm{P}($ Our result) & NP-hard [3] \\
$\ell>0$ & $\mathrm{P}[2]$ & $\mathrm{P}[1]$ \\
\hline
\end{tabular}

In this paper, we give a first polynomial-time algorithm for the undirected 2-DSPP with an arbitrary non-negative edge length function. That is, our algorithm works without assuming that each edge has a positive length.

Theorem 1.1. The undirected 2-DSPP with a non-negative edge length function can be solved in polynomial time.

We note that the polynomial solvability of the edge-disjoint variant of 2-DSPP is exactly the same as Table 1 , because the edge-disjoint case can be reduced to the vertex-disjoint case by considering the line graph with an appropriate length function.

We give a proof of Theorem 1.1 in Section 3. Before going to technical details, we here describe the outline of our algo- 
rithm. In the algorithm, we first consider the graph induced by the edges of length zero, and shrink its each connected component. Then, we obtain a graph with positive length edges. By replacing each edge with a pair of oppositely oriented arcs of the same length, we obtain a digraph with positive length arcs, and then apply a similar argument to [1]. A difficult point is how to deal with the vertices newly created by the shrinking operation. Since such a vertex corresponds to a subgraph, say $H$, of the original graph, we have to keep some information of $H$. Therefore, we solve the two disjoint paths problem in $H$ for every choice of terminal pairs with the aid of algorithms in $[8,9,10]$, and use the solution of this problem in our algorithm.

Note that Gottschau, Kaiser, and Waldmann [4] proved Theorem 1.1 based on the same arguments as ours, independently.

\section{Problem with Special Vertex Sets}

In this section, we introduce a variant of the two disjoint paths problem, which is used in our proof of Theorem 1.1. We show that this problem can be solved in polynomial time if the input satisfies a certain condition.

Let $G=(V, E)$ be a digraph and let $\mathcal{U}$ be a collection of disjoint vertex subsets of $V$ such that $G[U]$ is a complete digraph for each $U \in \mathcal{U}$, where $G[U]$ denotes the subgraph of $G$ induced by $U$. For notational convenience, we assume that a complete digraph contains a self-loop incident to each vertex. That is, the arc set $E_{U}$ of $G[U]$ is equal to $U \times U$. We say that a pair of two paths $P_{1}$ and $P_{2}$ in $G$ is $\mathcal{U}$-simple if, for each $i=1,2$ and for each $U \in \mathcal{U}$, one of the following holds:

- $P_{i}$ contains exactly two vertices in $U$ and an arc connecting them,

- $P_{i}$ contains exactly one vertex in $U$, or

- $P_{i}$ contains no vertex in $U$.

The intersection of $P_{i}$ and $U$ is represented by either an arc in $E_{U}$ or the emptyset as follows: it is represented by an arc connecting distinct vertices in the first case, by a self-loop in the second case, and by the emptyset in the third case.

For each $U \in \mathcal{U}$, suppose we are given a set $\mathcal{A}_{U} \subseteq\left(E_{U} \cup\{\emptyset\}\right)^{2}$ with $(\emptyset, \emptyset) \in \mathcal{A}_{U}$. For $U \in \mathcal{U}$, we say that a pair of two paths $P_{1}$ and $P_{2}$ in $G$ is admissible with respect to $\mathcal{A}_{U}$ if there exists a pair $\left(e_{1}, e_{2}\right) \in \mathcal{A}_{U}$ (possibly $e_{i}=\emptyset$ ) such that the intersection of $P_{i}$ and $U$ is represented by $e_{i} \in E_{U} \cup\{\emptyset\}$ for $i=1,2$. We say that a pair of two paths $P_{1}$ and $P_{2}$ in $G$ is admissible with respect to $\left\{\mathcal{A}_{U}\right\}_{U \in \mathcal{U}}$ if they are $\mathcal{U}$-simple and admissible with respect to $\mathcal{A}_{U}$ for every $U \in \mathcal{U}$. We now consider the following problem.

\section{Problem A}

Input. A digraph $G=(V, E)$ with a collection $\mathcal{U}$ of disjoint vertex subsets of $V$ such that $G[U]$ is a complete digraph for each $U \in \mathcal{U}$. We are also given a set $\mathcal{A}_{U} \subseteq\left(E_{U} \cup\{\emptyset\}\right)^{2}$ with $(\emptyset, \emptyset) \in \mathcal{A}_{U}$ for each $U \in \mathcal{U}$, two arc sets $E_{1}, E_{2} \subseteq$ $E \backslash \bigcup_{U \in \mathcal{U}} E_{U}$, and two pairs of vertices $\left(s_{1}, t_{1}\right)$ and $\left(s_{2}, t_{2}\right)$ in $G$.
Find. Disjoint paths $P_{1}$ and $P_{2}$ that are admissible with respect to $\left\{\mathcal{A}_{U}\right\}_{U \in \mathcal{U}}$ such that $P_{i}$ is a path from $s_{i}$ to $t_{i}$ and $E\left(P_{i}\right) \subseteq$ $E_{i} \cup \cup_{U \in \mathcal{U}} E_{U}$ for $i=1,2$, if they exist.

Note that, for a path $P, E(P)$ denotes the set of edges in $P$. Intuitively, this problem is to find two disjoint paths under the assumption that the possible routings in $U \in \mathcal{U}$ are known in advance, where $\mathcal{A}_{U}$ represents the possible combinations of the end points of the routings in $U$. The following theorem states that this problem can be solved in polynomial time if the digraph obtained from $G$ by shrinking all the components in $\mathcal{U}$ is acyclic, which plays an important role in our proof of Theorem 1.1. Here, shrinking a vertex set $U$ is an operation that replaces $U$ with a single vertex $u$ and removes all the self-loops incident to $u$.

Proposition 2.1. Suppose we are given a digraph $G=(V, E)$ with a collection $\mathcal{U}$ of disjoint vertex subsets of $V$ such that $G[U]$ is a complete digraph for each $U \in \mathcal{U}$. Assume that the digraph obtained from $G$ by shrinking each $U \in \mathcal{U}$ to a single vertex is acyclic. Then, Problem A in $G$ can be solved in polynomial time.

Proof. By adding new source vertices or new sink vertices if necessary, we may assume that there exist a unique arc $e_{s_{i}}$ leaving $s_{i}$ and a unique arc $e_{t_{i}}$ entering $t_{i}$ for $i=1,2$. Let $G^{\prime}=\left(V^{\prime}, E^{\prime}\right)$ be the acyclic digraph obtained from $G$ by shrinking $U$ to a single vertex for every $U \in \mathcal{U}$. Note that $E^{\prime}$ can be identified with $E \backslash \bigcup_{U \in \mathcal{U}} E_{U}$. We construct a new digraph $\mathcal{G}$ whose vertex set is $W:=E_{1} \times E_{2} \subseteq E^{\prime} \times E^{\prime}$ as follows. For an $\operatorname{arc} e$ in $G, \operatorname{head}_{G}(e)$ and tail $G_{G}(e)$ denote the head and the tail of $e$, respectively. For $\left(e_{1}, e_{2}\right),\left(e_{1}^{\prime}, e_{2}^{\prime}\right) \in W, \mathcal{G}$ has an arc from $\left(e_{1}, e_{2}\right)$ to $\left(e_{1}^{\prime}, e_{2}^{\prime}\right)$ if one of the following holds.

- $e_{1}^{\prime}=e_{1}, \operatorname{head}_{G^{\prime}}\left(e_{2}\right)=\operatorname{tail}_{G^{\prime}}\left(e_{2}^{\prime}\right)=: v$, and there is no path in $G^{\prime}$ from head $G_{G^{\prime}}\left(e_{1}\right)$ to $v$. Furthermore, if $v \in V^{\prime}$ is a vertex created by shrinking $U \in \mathcal{U}$, then $\left(\emptyset,\left(s_{2}^{\prime}, t_{2}^{\prime}\right)\right) \in \mathcal{A}_{U}$ holds, where $s_{2}^{\prime}:=\operatorname{head}_{G}\left(e_{2}\right)$ and $t_{2}^{\prime}:=\operatorname{tail}_{G}\left(e_{2}^{\prime}\right)$.

- $e_{2}^{\prime}=e_{2}, \operatorname{head}_{G^{\prime}}\left(e_{1}\right)=\operatorname{tail}_{G^{\prime}}\left(e_{1}^{\prime}\right)=: v$, and there is no path in $G^{\prime}$ from $\operatorname{head}_{G^{\prime}}\left(e_{2}\right)$ to $v$. Furthermore, if $v \in V^{\prime}$ is a vertex created by shrinking $U \in \mathcal{U}$, then $\left(\left(s_{1}^{\prime}, t_{1}^{\prime}\right), \emptyset\right) \in \mathcal{A}_{U}$ holds, where $s_{1}^{\prime}:=\operatorname{head}_{G}\left(e_{1}\right)$ and $t_{1}^{\prime}:=\operatorname{tail}_{G}\left(e_{1}^{\prime}\right)$.

- There exists a vertex $v \in V^{\prime}$ created by shrinking $U \in$ $\mathcal{U}$ such that $\operatorname{head}_{G^{\prime}}\left(e_{1}\right)=\operatorname{head}_{G^{\prime}}\left(e_{2}\right)=\operatorname{tail}_{G^{\prime}}\left(e_{1}^{\prime}\right)=$ $\operatorname{tail}_{G^{\prime}}\left(e_{2}^{\prime}\right)=: v$ and $\left(\left(s_{1}^{\prime}, t_{2}^{\prime}\right),\left(s_{2}^{\prime}, t_{2}^{\prime}\right)\right) \in \mathcal{A}_{U}$, where $s_{1}^{\prime}:=$ $\operatorname{head}_{G}\left(e_{1}\right), t_{1}^{\prime}:=\operatorname{tail}_{G}\left(e_{1}^{\prime}\right), s_{2}^{\prime}:=\operatorname{head}_{G}\left(e_{2}\right)$ and $t_{2}^{\prime}:=$ $\operatorname{tail}_{G}\left(e_{2}^{\prime}\right)$.

Then, we can see that Problem A has a solution if and only if $\mathcal{G}$ contains a path from $\left(e_{s_{1}}, e_{s_{2}}\right)$ to $\left(e_{t_{1}}, e_{t_{2}}\right)$ (see [1]). Since the size of $\mathcal{G}$ is bounded by a polynomial in $|E|$, Problem $\mathrm{A}$ in $G$ can be solved in polynomial time.

Note that the argument in this proof is essentially the same as $[1,3]$. 


\section{Proof of Theorem 1.1}

In this section, we give a proof of Theorem 1.1, that is, we show that the undirected 2-DSPP can be solved in polynomial time if the length of each edge is non-negative. Roughly, we shrink all the components induced by the edges of length zero, and then apply the arguments in [1].

We first show that the undirected 2-DSPP can be reduced to the following problem by shrinking all the edges of length zero.

\section{Problem B}

Input. A digraph $G=(V, E)$ with a collection $\mathcal{U}$ of disjoint vertex subsets of $V$ such that $G[U]$ is a complete digraph for each $U \in \mathcal{U}$. We are also given a set $\mathcal{A}_{U} \subseteq\left(E_{U} \cup\{\emptyset\}\right)^{2}$ with $(\emptyset, \emptyset) \in \mathcal{A}_{U}$ for each $U \in \mathcal{U}$, a length function $\ell$ : $E \rightarrow \mathbb{R}_{+}$, and two pairs of vertices $\left(s_{1}, t_{1}\right)$ and $\left(s_{2}, t_{2}\right)$ in $G$.

Find. Disjoint paths $P_{1}$ and $P_{2}$ that are admissible with respect to $\left\{\mathcal{A}_{U}\right\}_{U \in \mathcal{U}}$ such that $P_{i}$ is a shortest path from $s_{i}$ to $t_{i}$ for $i=1,2$, if they exist.

Lemma 3.1. The undirected 2-DSPP can be reduced to РRовLEM B in which $\ell(e)=0$ for any $e \in \bigcup_{U \in \mathcal{U}} E_{U}$ and $\ell(e)>0$ for any $e \in E \backslash \bigcup_{U \in \mathcal{U}} E_{U}$.

Proof. Suppose we are given an instance of the 2-DSPP in an undirected graph $G^{\circ}=\left(V, E^{\circ}\right)$. Consider the subgraph of $G^{\circ}$ consisting of all the edges of length zero, and let $\mathcal{U}$ be the collection of the vertex sets of its connected components that contain at least two vertices.

For each $U \in \mathcal{U}$, consider the subgraph $G^{\circ}[U]$ of $G^{\circ}$ induced by $U$. We remove all the edges of positive length in $G^{\circ}[U]$, since such edges cannot be used in shortest paths. Then, define $\mathcal{A}_{U} \subseteq\left(E_{U} \cup\{\emptyset\}\right)^{2}$ so that $\left(e_{1}, e_{2}\right) \in \mathcal{A}_{U}$ if and only if (1) $e_{1}=$ $\emptyset$, (2) $e_{2}=\emptyset$, or (3) $e_{1}=\left(s_{1}^{\prime}, t_{1}^{\prime}\right), e_{2}=\left(s_{2}^{\prime}, t_{2}^{\prime}\right)$, and $G^{\circ}[U]$ contains both an $s_{1}^{\prime}-t_{1}^{\prime}$ path and an $s_{2}^{\prime}-t_{2}^{\prime}$ path that are disjoint (each path might be a single vertex). Note that we can compute $\mathcal{A}_{U}$ by solving the two disjoint paths problem for each choice of $s_{1}^{\prime}, t_{1}^{\prime}, s_{2}^{\prime}$, and $t_{2}^{\prime}$, which can be done in polynomial time $[8,9$, 10].

Let $G=(V, E)$ be the digraph obtained from $G^{\circ}$ by replacing each edge with a pair of oppositely oriented arcs of the same length and by adding the arcs of length zero in $\bigcup_{U \in \mathcal{U}} E_{U}$ (see Figures 1 and 2). Then, we have that $\ell(e)=0$ for any $e \in$ $\bigcup_{U \in \mathcal{U}} E_{U}$ and $\ell(e)>0$ for any $e \in E \backslash \bigcup_{U \in \mathcal{U}} E_{U}$. We now show that the 2-DSPP in $G^{\circ}=\left(V, E^{\circ}\right)$ is equivalent to Problem B in $G=(V, E)$.

Suppose that there exist paths $P_{1}^{\circ}$ and $P_{2}^{\circ}$ that form a solution of the 2-DSPP in $G^{\circ}=\left(V, E^{\circ}\right)$. For $U \in \mathcal{U}$ and for $i=1,2$, the intersection of $P_{i}^{\circ}$ and $G^{\circ}[U]$ is a single connected component unless it is empty, because $P_{i}^{\circ}$ is a shortest $s_{i}-t_{i}$ path. If the intersection of $P_{i}^{\circ}$ and $G^{\circ}[U]$ forms a subpath connecting $s_{i}^{\prime} \in U$ and $t_{i}^{\prime} \in U$, then we replace it with an edge between $s_{i}^{\prime}$ and $t_{i}^{\prime}$. Then, by orienting all the edges in $P_{i}^{\circ}$ appropriately, we obtain an $s_{i}-t_{i}$ path $P_{i}$ for $i=1,2$. We can easily see that each $P_{i}$ is a shortest $s_{i}-t_{i}$ path, and $P_{1}$ and $P_{2}$ are admissible with respect to $\left\{\mathcal{A}_{U}\right\}_{U \in \mathcal{U}}$. Thus, there exists a solution of Problem B in $G=$ $(V, E)$.

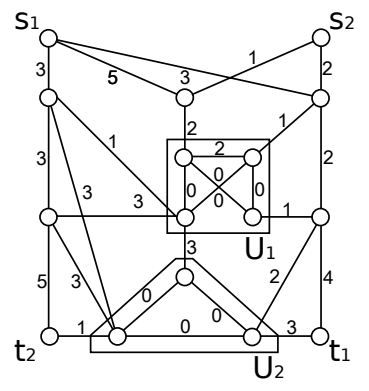

Figure 1: Graph $G^{\circ}=\left(V, E^{\circ}\right)$.

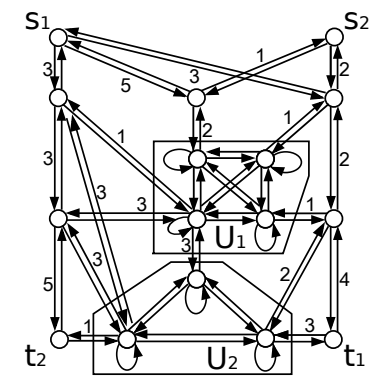

Figure 2: Construction of $G=(V, E)$.
Conversely, suppose that there exist paths $P_{1}$ and $P_{2}$ that form a solution of Рroblem B in $G=(V, E)$. We ignore the direction of the arcs. For each $U \in \mathcal{U}$ and for $i=1,2$, if $E\left(P_{i}\right) \cap E_{U}=\left(s_{i}^{\prime}, t_{i}^{\prime}\right)$, then we replace $\left(s_{i}^{\prime}, t_{i}^{\prime}\right)$ with an $s_{i}^{\prime}-t_{i}^{\prime}$ path in $G^{\circ}[U]$ based on the definition of $\mathcal{A}_{U}$. For example, if $E\left(P_{i}\right) \cap E_{U}=\left(s_{i}^{\prime}, t_{i}^{\prime}\right)$ for $i=1,2$, then $G^{\circ}[U]$ contains an $s_{1}^{\prime}-t_{1}^{\prime}$ path $Q_{1}$ and $s_{2}^{\prime}-t_{2}^{\prime}$ path $Q_{2}$ that are disjoint, and hence we replace an $\operatorname{arc}\left(s_{i}^{\prime}, t_{i}^{\prime}\right)$ with $Q_{i}$ for $i=1,2$. By applying this procedure for every $U \in \mathcal{U}$, we obtain an $s_{i}-t_{i}$ path $P_{i}^{\circ}$ in $G^{\circ}$ for $i=1,2$. We can easily see that each $P_{i}^{\circ}$ is a shortest $s_{i}-t_{i}$ path, and $P_{1}^{\circ}$ and $P_{2}^{\circ}$ are disjoint. Thus, there exists a solution of the 2-DSPP in $G^{\circ}=\left(V, E^{\circ}\right)$.

Therefore, the 2-DSPP in $G^{\circ}=\left(V, E^{\circ}\right)$ is equivalent to РвовLEM B in $G=(V, E)$.

In what follows, we consider an instance of Problem $\mathrm{B}$ in which $\ell(e)=0$ for any $e \in \bigcup_{U \in \mathcal{U}} E_{U}$ and $\ell(e)>0$ for any $e \in E \backslash \bigcup_{U \in \mathcal{U}} E_{U}$.

Let $G^{\prime}=\left(V^{\prime}, E^{\prime}\right)$ be the digraph obtained from $G$ by shrinking each $U \in \mathcal{U}$ to a single vertex (see Figure 3 ). Then, we can identify $E^{\prime}$ with $E \backslash \bigcup_{U \in \mathcal{U}} E_{U}$. For $i=1,2$, let $E_{i}$ be the set of edges in $E^{\prime}=E \backslash \bigcup_{U \in \mathcal{U}} E_{U}$ that are contained in some shortest $s_{i}-t_{i}$ path (see Figure 4). Note that $E_{i}$ can be computed in polynomial time by shortest path algorithms. Since $\ell(e)=0$ for any $e \in \bigcup_{U \in \mathcal{U}} E_{U}$, for $i=1$,2, we can observe that an $s_{i}-t_{i}$ path $P_{i}$ in $G$ is a shortest $s_{i}-t_{i}$ path if and only if $E\left(P_{i}\right) \backslash \bigcup_{U \in \mathcal{U}} E_{U} \subseteq E_{i}$.

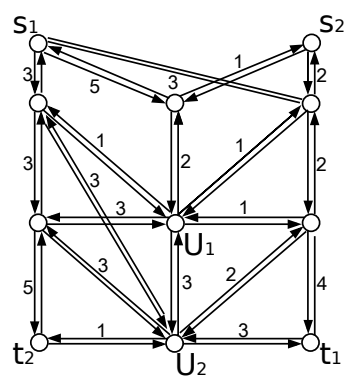

Figure 3: $G^{\prime}=\left(V^{\prime}, E^{\prime}\right)$.

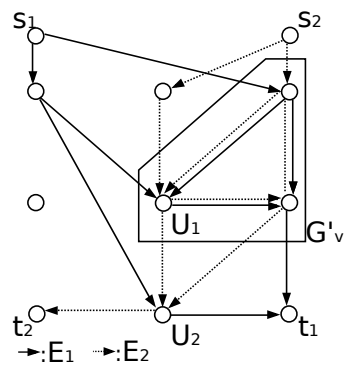

Figure 4: $E_{1}$ and $E_{2}$.
In order to apply Proposition 2.1, we construct an acyclic digraph in the same way as [1] as follows. Let $E_{0}=E_{1} \cap E_{2}$, $E_{1}^{*}=E_{1} \backslash E_{0}$, and $E_{2}^{*}=E_{2} \backslash E_{0}$. We remove all the arcs in $E^{\prime} \backslash\left(E_{1} \cup E_{2}\right)$ from $G^{\prime}$, contract all the arcs in $E_{0}$, and reverse all the arcs in $E_{2}^{*}$. Then, we obtain a digraph $G^{*}=\left(V^{*}, E^{*}\right)$. Let $V_{0} \subseteq V^{*}$ be the set of all the vertices in $V^{*}$ that are newly 


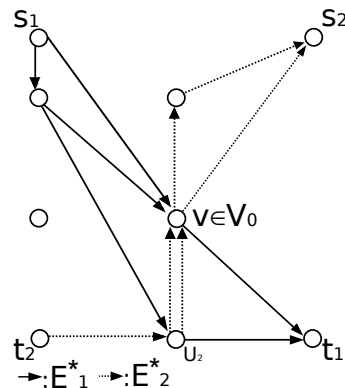

Figure 5: $G^{*}=\left(V^{*}, E^{*}\right)$.

created by contracting $E_{0}$. For $v \in V^{*}$, let $G_{v}^{\prime}$ be the subgraph of $G^{\prime}-\left(E^{\prime} \backslash\left(E_{1} \cup E_{2}\right)\right)$ induced by the vertex set corresponding to $v$. Note that $G_{v}^{\prime}$ is a single vertex for $v \in V^{*} \backslash V_{0}$. Then, it is shown in [1] that $G^{*}$ satisfies the following property.

Lemma 3.2 (Bérczi and Kobayashi [1]). Let $E_{0}, E_{1}, E_{2}, E_{1}^{*}$, $E_{2}^{*}, G^{*}$, and $V_{0}$ be defined as above. Then, we have the following.

(1) For each $v \in V_{0}, G_{v}$ is an acycilic digraph whose arcs are in $E_{1} \cap E_{2}$.

(2) $G^{*}$ is an acyclic digraph.

By using this lemma, we show the following.

Lemma 3.3. There exists a polynomial-time algorithm for Problem B in which $\ell(e)=0$ for any $e \in \bigcup_{U \in \mathcal{U}} E_{U}$ and $\ell(e)>0$ for any $e \in E \backslash \bigcup_{U \in \mathcal{U}} E_{U}$.

Proof. Let $E_{0}, E_{1}, E_{2}, E_{1}^{*}, E_{2}^{*}, G^{*}$, and $V_{0}$ be defined as above. For $v \in V^{*}$, let $G_{v}$ be the digraph obtained from $G_{v}^{\prime}=\left(V_{v}^{\prime}, E_{v}^{\prime}\right)$ by expanding all the vertices corresponding to the elements in $\mathcal{U}$. By abuse of notation, the restriction of $\mathcal{U}$ to $G_{v}$ is also denoted by $\mathcal{U}$. Let $U_{v}$ be the set of vertices in $G$ corresponding to $v$ for each $v \in V^{*}$, and define $\mathcal{U}^{\prime}=\left\{U_{v}\left|v \in V^{*},\right| U_{v} \mid \geq 2\right\}$. Then, for each $v \in V^{*}$ with $\left|U_{v}\right| \geq 2$, define $\mathcal{A}_{U_{v}}^{\prime} \subseteq\left(E_{U_{v}} \cup\{\emptyset\}\right)^{2}$ so that $\left(e_{1}, e_{2}\right) \in \mathcal{A}_{U_{v}}^{\prime}$ if and only if one of the following holds:

- $\left(e_{1}, e_{2}\right)=(\emptyset, \emptyset)$.

- $e_{1}=\left(s_{1}^{\prime}, t_{1}^{\prime}\right), e_{2}=\emptyset$, and $G_{v}$ contains an $s_{1}^{\prime}-t_{1}^{\prime}$ path $P_{1}$ such that $P_{1}$ and $P_{2}:=\emptyset$ are admissible with respect to $\left\{A_{U}\right\}_{U \in \mathcal{U}}$.

- $e_{1}=\emptyset, e_{2}=\left(t_{2}^{\prime}, s_{2}^{\prime}\right)$, and $G_{v}$ contains an $s_{2}^{\prime}-t_{2}^{\prime}$ path $P_{2}$ such that $P_{1}:=\emptyset$ and $P_{2}$ are admissible with respect to $\left\{A_{U}\right\}_{U \in \mathcal{U}}$.

- $e_{1}=\left(s_{1}^{\prime}, t_{1}^{\prime}\right), e_{2}=\left(t_{2}^{\prime}, s_{2}^{\prime}\right)$, and $G_{v}$ contains disjoint paths $P_{1}$ and $P_{2}$ that are admissible with respect to $\left\{\mathcal{A}_{U}\right\}_{U \in \mathcal{U}}$ such that $P_{i}$ is a path from $s_{i}^{\prime}$ to $t_{i}^{\prime}$ for $i=1,2$.

Note that $\mathcal{A}_{U_{v}}^{\prime}$ can be computed in polynomial time by Proposition 2.1 and Lemma 3.2 (1).

Consider the digraph obtained from $G^{*}$ by expanding each vertex $v \in V^{*}$ with $\left|U_{v}\right| \geq 2$ to $U_{v}$ and by adding edges so that each $U_{v}$ induces a complete digraph. Then, the edge set of the obtained digraph can be identified with $E_{1}^{*} \cup E_{2}^{*} \cup \cup_{U \in \mathcal{U}^{\prime}} E_{U}$.
In this digraph, we find disjoint paths $P_{1}^{\prime}$ and $P_{2}^{\prime}$ that are admissible with respect to $\left\{\mathcal{A}_{U}^{\prime}\right\}_{U \in \mathcal{U}^{\prime}}$ such that $P_{1}^{\prime}$ is a path from $s_{1}$ to $t_{1}, P_{2}^{\prime}$ is a path from $t_{2}$ to $s_{2}$, and and $E\left(P_{i}^{\prime}\right) \subseteq E_{i}^{*} \cup \bigcup_{U \in \mathcal{U}^{\prime}} E_{U}$ for $i=1,2$. This can be done in polynomial time by Proposition 2.1 and Lemma 3.2 (2). Note that $P_{2}^{\prime}$ is a path from $t_{2}$ to $s_{2}$ since $E_{2}^{*}$ is obtained from $E_{2} \backslash E_{0}$ by reversing all the arcs. By the definition of $\mathcal{A}_{U_{v}}^{\prime}, P_{1}^{\prime}$ and $P_{2}^{\prime}$ correspond to disjoint paths $P_{1}$ and $P_{2}$ in $G$ that are admissible with respect to $\left\{\mathcal{A}_{U}\right\}_{U \in \mathcal{U}}$ such that $E\left(P_{i}\right) \backslash \bigcup_{U \in \mathcal{U}} E_{U} \subseteq E_{i}$ for $i=1$,2, which form a solution of Problem B.

By Lemmas 3.1 and 3.3, we obtain Theorem 1.1.

\section{Acknowledgement}

The authors thank the anonymous reviewer for helpful comments. They also thank Gianpaolo Oriolo and Jannik Matuschke for handling our paper and [4] appropriately. This work is supported by JST ACT-I Grant Number JPMJPR17UB and by JSPS KAKENHI Grant Numbers JP16K16010 and JP16H03118.

\section{References}

[1] Kristóf Bérczi and Yusuke Kobayashi. The directed disjoint shortest paths problem. In Proceedings of the 25th Annual European Symposium on Algorithms (ESA 2017), pages 13:1-13:13, 2017.

[2] Tali Eilam-Tzoreff. The disjoint shortest paths problem. Discrete Applied Mathematics, 85(2):113-138, 1998.

[3] Steven Fortune, John E. Hopcroft, and James Wyllie. The directed subgraph homeomorphism problem. Theoretical Computer Science, 10:111121, 1980.

[4] Marinus Gottschau, Marcus Kaiser, and Clara Waldmann. The undirected two disjoint shortest paths problem. arXiv:1809.03820, 2018.

[5] Richard M. Karp. On the computational complexity of combinatorial problems. Networks, 5:45-68, 1975.

[6] James F. Lynch. The equivalence of theorem proving and the interconnection problem. SIGDA Newsletter, 5(3):31-36, 1975

[7] Neil Robertson and Paul D. Seymour. Graph minors. XIII. The disjoint paths problem. Journal of Combinatorial Theory, Series B, 63(1):65-110, 1995.

[8] Paul D. Seymour. Disjoint paths in graphs. Discrete Mathematics, 29:293-309, 1980.

[9] Yossi Shiloach. A polynomial solution to the undirected two paths problem. Journal of the ACM, 27(3):445-456, 1980.

[10] Carsten Thomassen. 2-linked graphs. European Journal of Combinatorics, 1:371-378, 1980. 